\title{
Topology and Shape Preserving Parallel Thinning for 3D Digital Images - A New Approach
}

\author{
P.K. Saha and D. Dutta Majumder \\ Electronics and Communication Sciences Unit \\ Indian Statistical Institute, 203 Barrackpur Trunk Road \\ Calcutta 700035 INDIA
}

\begin{abstract}
This paper is concerned with a new parallel thinning approach for three dimensional (3D) digital images that preserves the topology and maintains their shape. We introduce a new approach of selecting shape points and outer-layer used for erosion during each iteration. The approach produces good skeleton for different types of corners. The concept of using two image versions in thinning is introduced and its necessity in parallel thinning is justified. The robustness of the algorithm under pseudo random noise with respect to shape properties is studied and the results are found to be satisfactory.
\end{abstract}

\section{Introduction}

The processing of $3 \mathrm{D}$ digital images has become of increasing interest with the rapid growth of $3 \mathrm{D}$ image analysis and computer vision based applications in different fields such as astrophysics, geophysics, industrial inspection and medical imaging. Of all these $3 \mathrm{D}$ medical imaging is the most popular one such as Computed Tomography (CT), Magnetic Resonance Imaging (MRI), Ultrasound Echography (UE), Positron Emission Tomography (PET), Digital Subtraction Angiography (DSA) etc. This paper is concerned with a new parallel thinning approach for three dimensional (3D) digital images. Many image processing methodologies such as smoothing, filtering, thinning and segmentation are of interest in various applications to $3 \mathrm{D}$ image processing. The objective of $3 \mathrm{D}$ image thinning is to produce a medial surface representation that preserves the topology and maintains the shape of an object as much as possible. Thinning makes a compact representation of an object and hence is computationally attractive for future analysis.

One of the important uses of thinning is to decompose an object into meaningful segments [1]. Although $3 \mathrm{D}$ thinning has a lot of interest in $3 \mathrm{D}$ image processing [2], only a few publications $[3,4,5,6,7,8,2]$ are found in $3 D$ thinning. Unfortunately none of the previous researchers studied the behavior of their algorithms around different types of corners. Also they did not study the robustness of their algorithms under noise. In this paper we consider the aspects of thinning algorithm stressing its behavior and robustness under pseudo random noise. 
Theoretical aspect of the proposed thinning approach that produces a medial surface representation of 3D object is described in Section 2. The parallel thinning algorithm is described and the experimental results are presented in Sections 3 and 4 respectively.

\section{The Thinning Approach}

We consider thinning as an approach of producing a medial surface representation of a 3D digital object that preserves the topology and maintains the shape of the object to the maximum extent. The proposed thinning approach is an iterative erosion process that consists of two steps namely primary-thinning and final-thinning. The results of these steps are called primary-skeleton and finalskeleton respectively.

Our thinning approach exploits the information from two versions of an image implicitly stored throughout the thinning procedure. One image version denotes the black/white configuration before the current iteration while the other denotes the current stage of the processed image. Here, it is worthy to mention that simple points $[9,10]$ are always detected on the current version of the image while the shape preserving constraints are mostly defined on the image version before each current iteration. This idea is quite different from other works $[8,4]$ where only one version of image is used for thinning. At this point it should be made clear that in this work, iteration and scan are two completely different concepts. A scan is a (point by point) traversal of the entire image when subjected to the thinning process. On the other hand, an iteration is completed after considering the entire outer-layer of an object through proper topology and shape constraints. An iteration may consist of one or more scans in which case the operation in each scan is generally different. The set of points considered for erosion during an iteration defines the outer-layer for that iteration. Before we describe the thinning procedure let us present some definitions and conditions in this context. In this paper we follow conventional definitions $[10,1]$ of adjacency, path, connectivity etc.

Definition 1. $\mathcal{N}(p)$ denotes the set of 27 points in the $3 \times 3 \times 3$ neighborhood of p. An s-point of $\mathcal{N}(p)$ is 6-adjacent to $p$. An e-point of $\mathcal{N}(p)$ is 18-adjacent but not 6 -adjacent to $p$. A v-point of $\mathcal{N}(p)$ is 26 -adjacent but not 18-adjacent to $p$. Two $s$ points $a, b \in \mathcal{N}(p)$ are called opposite if they are not 26-adjacent. Otherwise, they are called non-opposite s-points. Let $a, b, c$ denote three non-opposite s-points of $\mathcal{N}(p)$. Five points are defined with respect to $a, b, c, p$ as follows: $(1) e(a, b, p)$ is the point $q$ such that $q \in \mathcal{N}^{*}(p)$ and 6-adjacent to $a, b,(2) v(a, b, c, p)$ is the point $q$ such that $q \in \mathcal{N}^{*}(p)$ and 6-adjacent to $e(a, b, p), e(b, c, p), e(c, a, p),(3) f_{1}(a, p)$ is the point $q$ such that $q \notin \mathcal{N}(p)$ and 6-adjacent to $a,(4) f_{2}(a, b, p)$ is the point $q$ such that $q \notin \mathcal{N}(p)$ and 6-adjacent to $f_{1}(a, p), e(a, b, p)$ and $(4) f_{3}(a, b, p)$ is the point $q$ such that $q \notin \mathcal{N}(p)$ and 6-adjacent to $f_{2}(a, b, p), f_{2}(b, a, p)$. Let $p=(i, j, k)$ be a point. The set $\{(i-1, j, k),(i, j-1, k)(l, j, k-1)\}$ of three non-opposite $s$-point of $p$ is denoted as $T(p)$. Thus $T(p)$ contains the s-points of $\mathcal{N}(p)$ which are at the west, south and bottom sides of $p$. 
In the following definitions and conditions $(a, d),(b, e)$ and $(c, f)$ denote three distinct unordered pairs of opposite $s$-points of $\mathcal{N}(p)$ unless stated otherwise.

Definition 2. Let condition $C_{1}$ be ' $x \in\{b, e, c, f\}$ '; condition $C_{2}$ be ' $x, y \in$ $\{b, e, c, f\}$ and $x, y$ are non-opposite'; condition $C_{3}$ be ' $x \in\{b, e, c, f\}$ and $x \in$ $T(p)^{\prime} ;$ condition $C_{4}$ be ' $x, y \in\{b, e, c, f\} ; x, y$ are non-opposite and $x \in T(p)^{\prime}$ and condition $C_{5}$ be ' $x, y \in\{b, e, c, f\} ; x, y$ are non-opposite and $x, y \in T(p)^{\prime}$ '. We define a middle plane and an extended middle plane of $\mathcal{N}(p)$ as follows: $\mathcal{M}(a, d, p)=\left\{x \mid C_{1}\right\} \cup\left\{e(x, y, p) \mid C_{2}\right\}$, $\mathcal{E} \mathcal{M}(a, d, p)=\mathcal{M}(a, d, p) \cup\left\{f_{1}(x, p) \mid C_{3}\right\} \cup\left\{f_{2}(x, y, p) \mid C_{4}\right\} \cup\left\{f_{3}(x, y, p) \mid C_{5}\right\}$.

Definition 3. During an iteration a black point $p$ is an s-open point if at least one $s$-point of $\mathcal{N}(p)$ is white before the iteration. During an iteration a black point $p$ is an e-open point if $p$ is not an $s$-open point and an e-point $e(a, b, p)$ is white while the points $f_{1}(a, p), f_{1}(b, p)$ are black before the iteration. During an iteration a black point $p$ is a $v$-open point if $p$ is neither an s-open point nor an $e$-open point and a $v$-point $v(a, b, c, p)$ is white while the points $f_{1}(a, p)$, $f_{1}(b, p), f_{1}(c, p)$ are black before the iteration. The set of $s$-open, e-open and $v$-open points defines the outer-layer in an iteration. It is understood from the above definitions that the labeling of points as $s$-open, $e$-open and $v$-open points is made once before each iteration.

Condition 4. During an iteration a point $p$ satisfies Condition 4 if there exist two opposite s-points $a, d \in \mathcal{N}(p)$ such that $\mathcal{E} \mathcal{M}(a, d, p)$ contains a 6-closed path of white points encircling $p$ and each of surface $(a, p)$ and surface $(d, p)$ contains at least one black point before the iteration.

Condition 5. During an iteration a point $p$ satisfies Condition 5 if there exists a pair of opposite $s$-points $(a, d)$ such that $d \in T(p)$, $a$ is white, $d$ or $f_{1}(d, p)$ is white and each of the sets $\{e(a, b, p), b, e(b, d, p)\},\{e(a, c, p), c, e(c, d, p)\}$, $\{e(a, e, p), e, e(d, e, p)\},\{e(a, f, p), f, e(d, f, p)\},\{v(a, b, c, p), e(b, c, p), v(b, c$, $d, p)\},\{v(a, b, f, p), e(b, f, p), v(b, d, f, p)\},\{v(a, c, e, p), e(c, e, p), v(c, d, e, p)\}$, $\{v(a, e, f, p), e(e, f, p), v(d, e, f, p)\}$ contains at least one black point before the iteration.

Condition 6. During an iteration a point $p$ satisfies Condition 6 if for each middle plane $\mathcal{M}(a, d, p)$ of $\mathcal{N}(p)$ - either all $e$-points in $\mathcal{M}(a, d, p)$ are black before the iteration or the current black points of $\mathcal{M}(a, d, p)$ generate single 26component without any tunnel $[10,1]$.

Definition 7. During an iteration a function is defined on the black/white configuration before the iteration as follows:

$$
\text { thick }(a, d, p)=\left\{\begin{array}{l}
\text { true if } a \text { and } f_{1}(d, p) \text { are white while } d \text { is black, } \\
\text { false otherwise. }
\end{array}\right.
$$

Condition 8. A point $p$ satisfies Condition 8 if $\operatorname{thick}(a, d, p)$, where $d \in T(p)$, is true and the current black points of each of $\mathcal{M}(b, e, p)$ and $\mathcal{M}(c, f, p)$ generate single 26-component without any tunnel. 
Condition 9. A point $p$ satisfies Condition 9 if $\operatorname{thick}(a, d, p)$ and thick $(b, e, p)$, where $d, e \in T(p)$, are true and the current black points of $\mathcal{M}(c, f, p)$ generate single 26-component without any tunnel.

Condition 10. A point $p$ satisfies Condition 10 if $\operatorname{thick}(a, d, p)$, thick $(b, e, p)$ and thick $(c, f, p)$, where $d, e, f \in T(p)$, are true.

Definition 11. During an iteration a black point is an erodable point if it is a simple point and satisfies any of the Conditions 8,9 or 10 .

\subsection{Primary-Thinning}

As mentioned earlier primary-thinning is an iterative procedure and iterations are continued as long as any point is deleted in the last iteration. Each iteration is completed in three successive scans. During the first scan an unmarked s-open point is marked if it is a shape point. When it is not a shape point, it is deleted if it is a simple point, otherwise it is left unmarked. During the second scan an unmarked $e$-open point is deleted if it is a simple point and satisfies Condition 6 . During the third scan an unmarked $v$-open point is deleted if it is a simple point.

\section{$2.2 \quad$ Final-Thinning}

From the definition of shape point it may be understood that a two-point thick slanted surface may occur in primary-skeleton. Final-thinning is necessary to get a proper skeleton for such cases. This is a single iteration procedure and the iteration consists of single scan. During this scan a black point $p$ (irrespective of whether $p$ is marked or unmarked) is deleted if it is an erodable point.

\section{The Parallel Thinning Approach}

In this section we describe a parallel thinning algorithm based on the approach discussed in Section 2. For parallelization we use the concept of sub-fields $[3,4]$. An image is partitioned into eight disjoint subsets such that no two members $p, q$ in the same subset are 26-adjacent. The members of each subset may be used for parallel erosion. Eight subsets $O_{0}, O_{1}, \cdots O_{7}$ are defined as follows:

$$
\begin{array}{r}
O_{l}=\{(2 \times i+f, 2 \times j+g, 2 \times k+h) \mid i, j, k=0, \pm 1, \pm 2, \cdots ; \\
\left.f, g, h \in\{0,1\} \text { and } 2^{2} \times f+2^{1} \times g+2^{0} \times h=l\right\}
\end{array}
$$

such that two points $p, q \in O_{l}$ are never 26-adjacent. Each scan of the thinning algorithm may be completed in eight cycles and at $l$ th cycle the image subset $O_{l}$ is subjected to parallel erosion. The parallel algorithm requires $\mathrm{m}^{3} / 8$ processors for an image of size $m \times m \times m$ and it needs 8 cycles to complete each scan. The image size means the size of the smallest rectangular parallelepiped that encloses the set of black points. 


\section{Results and Discussion}

To test the effectiveness of the proposed algorithm it is applied on several $3 \mathrm{D}$ objects. We present three of these results in Figures 1-3. In all these figures backgrounds are made dark to render a better visual effect. Both noiseless and noisy versions (generated in a pseudo random manner) are shown. The surface representation of both noiseless and noisy versions of Figures 1-3 are visually satisfactory.

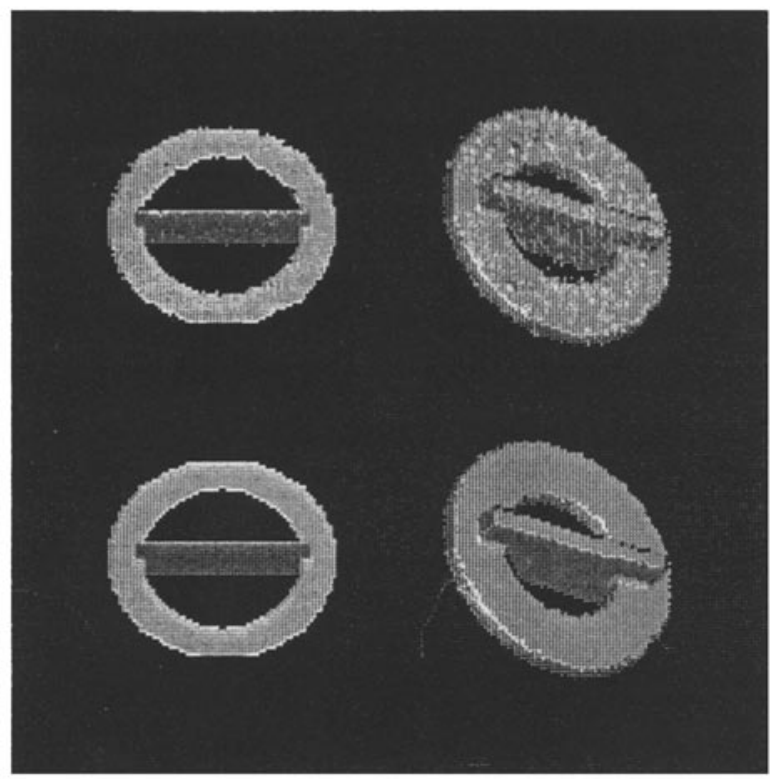

Fig. 1. Results of thinning. Top row (from left to right): original object and skeleton. Bottom row (from left to right): original object with noise and skeleton.

A new parallel thinning algorithm of $3 \mathrm{D}$ digital images with topology and shape preserving properties has been developed in this chapter. To preserve topology we have applied the concept of simple points $[9,10]$. On the other hand the concept of sub-fields [3] has been used for parallel implementation. The concepts of open points and shape points have been introduced and applied to $3 \mathrm{D}$ thinning. The concept of open points produces proper skeleton around different types of corners has been justified. Also, the shape points are found to be robust under noise. We have used two versions of the image - one before the current iteration while the other being the currently processed image. This concept has 


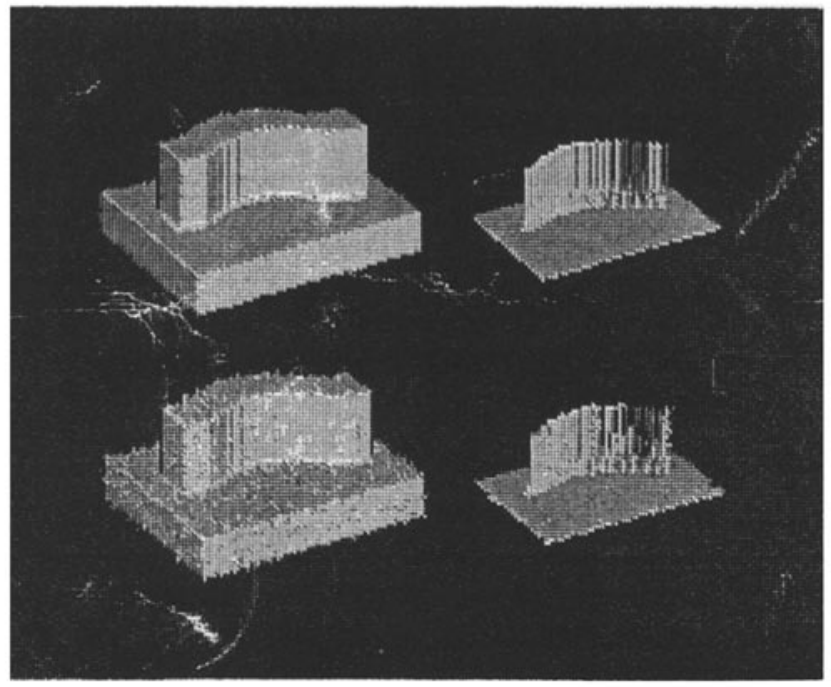

Fig. 2. Results of thinning. Top row (from left to right): original object and skeleton. Bottom row (from left to right): original object with noise and skeleton.

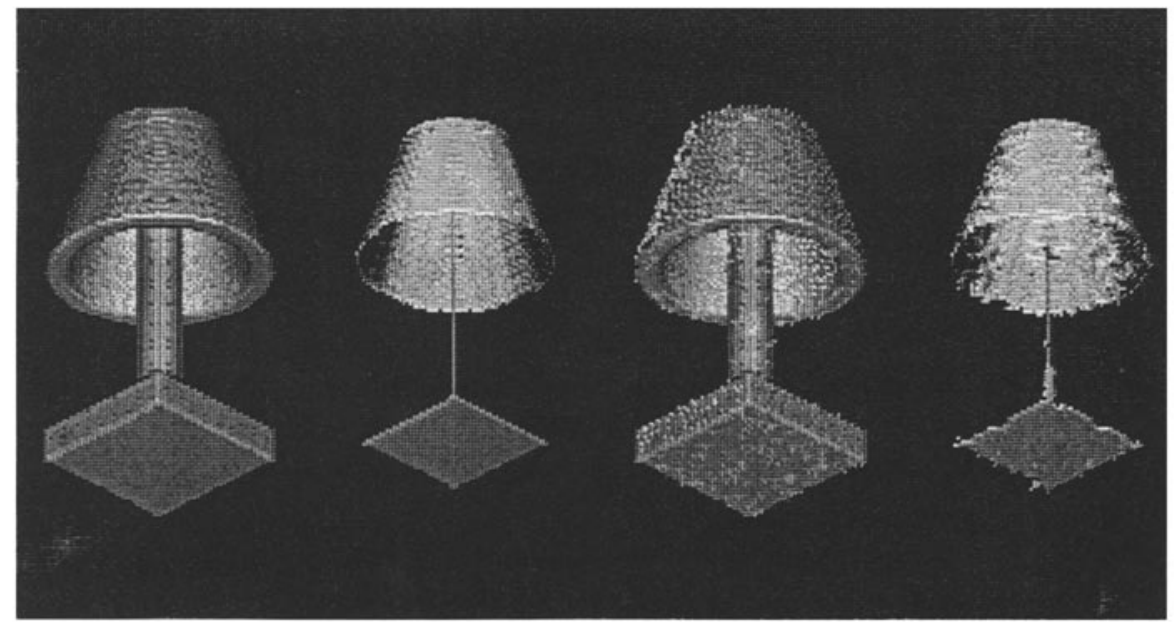

Fig. 3. Results of thinning. From left to right: original object, skeleton, original object with noise and skeleton. 
made a major improvement in the quality of thinned image. The results of application of the parallel thinning algorithm on several synthetically generated $3 \mathrm{D}$ objects and their noisy versions have been presented.

\section{References}

1. Saha, P. K., Chaudhuri, B. B.: 3D Digital topology under binary transformation with applications. CVGIP: Image Understanding 63 (1996) 418-429

2. Ma, C. M: On topology preservation in 3D thinning. CVGIP: Image Understanding 59 (1994) 328-339

3. Golay, M. J.: Hexagonal Parallel Pattern Transformations. IEEE Trans. Comput. C-18 (1969) 733-740

4. Hafford, K. J., Preston Jr, K.: Three-dimensional Skeletonization of Elongated Solids. Comput. Vision Graphics Image Process. 27 (1984) 78-91

5. Lobregt, S., Verbeek, P. W., Groen, F. C. A.: Three-dimensional skeletonization: principle and algorithm. IEEE Trans. Pattern Anal. Mach. Intell. PAMI-2 (1980) 75-77

6. Mukherjee, J, Das, P. P., Chatterjee, B. N.: Thinning of 3-D images using the Safe Point Thinning Algorithm (SPTA). Pattern Recognition Letters 10 (1989) 167-173

7. Srihari, S. N.: Representation of three-dimensional digital images. ACM Comput. Surveys 13 (1981) 400-424

8. Tsao, Y. F., Fu, K. S.: A parallel thinning algorithm for 3D pictures. Comput. Graphics Image Process. 17 (1981) 315-331

9. Saha, P. K., Chaudhuri, B. B., Chanda, B., DuttaMajumder, D.: Topology preservation in 3D digital space. Pattern Recognition 27 (1994) 295-300

10. Saha, P. K., Chaudhuri, B. B.: Detection of 3-D simple points for topology preserving transformations with application to thinning. IEEE Trans. on Pattern Anal. Mach. Intell. 16 (1994) 1028-1032 